\title{
非对称陀螺自由基分子光谱的标识分析 ${ }^{*}$ \\ $-\mathrm{NO}_{2}$ 基振动态纯转动光谱分析
}

\author{
刘显炎 ${ }^{* *}$ 刘效庸 刘红平郭远清 黄光明 \\ 林洁丽高晖 段传喜 \\ (中国科学院武汉物理与数学研究所, 波谱与原子分子物理国家重点实验室, 武汉 430071)
}

\begin{abstract}
摘要系统阐述了非对称陀螺分子光谱的标识分析工作涉及的一些关键理论问题, 包括有效 Hamilton 量、表象和基矢、能级识别、跃迁选择规则、相对强度和 Zeeman 调制率等, 并在有效 Hamilton 量中引入高阶离心畸变项，大大提高了计算精度，并使高 转动量子数跃迁的标识分析成为可能. 作为应用实例, 对 ${ }^{14} \mathrm{~N}^{16} \mathrm{O}_{2}$ 基振动态的零场和磁 共振谱进行了统一分析(global analysis), 获得了迄今为止最精确、最完整的 ${ }^{14} \mathrm{~N}^{16} \mathrm{O}_{2}$ 基振 动态分子结构参数, 并对 Curl 和 Brown 等人过去未能标识的 ${ }^{14} \mathrm{~N}^{16} \mathrm{O}_{2}$ 高转动量子数跃迁 远红外激光磁共振谱线进行了成功标识.
\end{abstract}

\section{关键词 非对称陀螺自由基 高阶离心畸变 ${ }^{14} \mathrm{~N}^{16} \mathrm{O}_{2}$ 基振动态光谱的统一分析}

自然界许多具有特殊意义的分子如 $\mathrm{PO}_{2}, \mathrm{NO}_{2}, \mathrm{CH}_{2} \mathrm{Br}, \mathrm{CH}_{2} \mathrm{~F}$ 等都是非对称陀螺自由基分 子. 正因为如此, 对非对称陀螺自由基分子光谱进行研究, 获得对这类分子的结构特性的深人 了解，具有显而易见的科学和现实意义，是分子光谱学的重要任务之一.

对分子的实验谱线进行标识分析是了解分子的内部结构信息的必经之路. 但是非对称陀 螺分子作为一种多原子分子, 其光谱结构十分复杂. 尤其是非对称陀螺自由基分子的磁共振 谱, 由于 Zeeman 效应的卷人, 较之其在零场条件下的光谱更为复杂 ${ }^{[1]}$. 如 $\mathrm{CH}_{2} \mathrm{Br}$ 和 $\mathrm{CH}_{2} \mathrm{~F}$ 等分 子的部分远红外激光磁共振迄今仍未获得圆满标识 ${ }^{[2,3]}$. 对非对称陀螺分子光谱进行标识分析 历来是分子光谱学中的难点之一, 是一项具有重要意义而又极富挑战性的工作.

Sears 对非对称陀螺自由基分子光谱的标识分析曾做过系统的研究, 并建立了一套比较完 善的、用于非对称陀螺自由基分子光谱标识分析的计算程序 ${ }^{[1,4]}$. Sears 程序曾被广泛应用于非 对称陀螺自由基分子如 $\mathrm{NH}_{2}, \mathrm{CH}_{2}, \mathrm{HCO}, \mathrm{CH}_{2} \mathrm{~F}$ 等光谱的计算, 并获得了相当大的成功. 但是由 于 Sears 程序中采用的有效 Hamilton 量不够完备, 没有考虑分子转动高阶离心畸变项以及电子 自旋-分子转动高阶离心畸变项的贡献, 对于一些离心畸变严重的非对称陀螺分子 (如 $\mathrm{H}_{2} \mathrm{O}$, $\mathrm{NO}_{2}$ 等)尤其是这些分子的高转动量子数跃迁往往不能进行有效的处理.

我们将高阶离心畸变项引人非对称陀螺分子的有效 Hamilton 量, 并设计了相应的

2000-01-19 收稿, 2000-07-17 收修改稿

* 国家自然科学基金(批准号: 19704016)和“九五”攀登计划资助项目

** E-mail: frankxliu@sina.com, yyl@nmr.whcnc.ac.cn 
Fortran77 计算程序. 利用该程序成功地标识分析了所有 $\mathrm{NO}_{2}$ 基振动态的纯转动谱. 本文详细 报道了这一工作.

\section{1 有效 Hamilton 量}

分子能级的计算须借助有效 Hamilton 量. 本文采用 $I^{r}$ 表象, 即固定于分子的坐标轴 $x, y, z$ 分别与分子的惯量主轴 $b, c, a$ 对应 ${ }^{[5]}$. 有效 Hamilton 量具有如下形式:

$$
H=H_{W}+H_{F S}+H_{H F S}+H_{Z} \text {. }
$$

这里 Watson 型 $A$ 约化 Hamilton 量 $^{[6]}$

$$
H_{W}=H_{R}+H_{C D}
$$

其中转动 Hamilton 量

$$
H_{R}=A N_{z}^{2}+B N_{x}^{2}+C N_{y}^{2}=\frac{B+C}{2} N^{2}+\left(A-\frac{B+C}{2}\right) N_{z}^{2}+\frac{B-C}{2} N_{x y}^{2},
$$

分子转动离心畸变 Hamilton 量

$$
\begin{aligned}
H_{C D}= & -\Delta_{N} N^{4}-\Delta_{N K} N^{2} N_{z}^{2}-\Delta_{K} N_{z}^{4}-2 \delta_{N} N^{2} N_{x y}^{2}-\delta_{K}\left\{N_{z}^{2}, N_{x y}^{2}\right\} \\
& +H_{N} N^{6}+H_{N K} N^{4} N_{z}^{2}+H_{K N} N^{2} N_{z}^{4}+H_{K} N_{z}^{6} \\
& +2 h_{N} N^{4} N_{x y}^{2}+h_{N K} N^{2}\left\{N_{z}^{2}, N_{x y}^{2}\right\}+h_{K}\left\{N_{z}^{4}, N_{x y}^{2}\right\} \\
& +L_{N} N^{8}+L_{N N K} N^{6} N_{z}^{2}+L_{N K} N^{4} N_{z}^{4}+L_{K K N} N^{2} N_{z}^{6}+L_{K} N_{z}^{8} \\
& +2 l_{N} N^{6} N_{x y}^{2}+l_{N K} N^{4}\left\{N_{z}^{2}, N_{x y}^{2}\right\}+l_{K N} N^{2}\left\{N_{z}^{4}, N_{x y}^{2}\right\}+l_{K}\left\{N_{z}^{6}, N_{x y}^{2}\right\} \\
& +P_{N} N^{10}+P_{N N K} N^{8} N_{z}^{2}+P_{N K} N^{6} N_{z}^{4}+P_{K N} N^{4} N_{z}^{6}+P_{K K N} N^{2} N_{z}^{8}+P_{K} N_{z}^{10} \\
& +2 p_{N} N^{8} N_{x y}^{2}+p_{N N K} N^{6}\left\{N_{z}^{2}, N_{x y}^{2}\right\}+p_{K K N} N^{2}\left\{N_{z}^{6}, N_{x y}^{2}\right\}+p_{K}\left\{N_{z}^{8}, N_{x y}^{2}\right\} \\
& +Q_{N N K} N^{8} N_{z}^{4}+Q_{K K N} N^{4} N_{z}^{8}+Q_{K K K N} N^{2} N_{z}^{10}+Q_{K} N_{z}^{12},
\end{aligned}
$$

式中 $N_{x y}^{2}=N_{x}^{2}-N_{y}^{2}, \quad\{A, B\}=A B+B A$.

精细结构 Hamilton 量

$$
H_{F S}=H_{S R}+H_{S R C D}+H_{S S},
$$

其中电子自旋-分子转动相互作用项

$$
H_{S R}=\frac{1}{2} \sum_{k=0}^{2}{ }^{\prime}\left[T^{k}(\varepsilon) \cdot T^{k}(N, S)+T^{k}(N, S) \cdot T^{k}(\varepsilon)\right],
$$

电子自旋-分子转动离心畸变项

$$
\begin{aligned}
H_{S R C D}= & \Delta_{N}^{S} N^{2}(N \cdot S)+\frac{1}{2} \Delta_{N K}^{S}\left\{N^{2}, N_{z} S_{z}\right\}+\Delta_{K N}^{S} N_{z}^{2} N \cdot S+\Delta_{K}^{S} N_{z}^{3} S_{z} \\
& +\delta_{N}^{S} N_{x y}^{2} N \cdot S+\frac{1}{2} \delta_{K}^{S}\left\{N_{x y}^{2}, N_{z} S_{z}\right\}+H_{K}^{S} N_{z}^{5} S_{z}+L_{K}^{S} N_{z}^{7} S_{z},
\end{aligned}
$$

电子自旋偶极相互作用项(仅当 $S>1 / 2$ 时考虑)

$$
H_{S S}=\frac{D}{3}\left(2 S_{z}^{2}-S_{x}^{2}-S_{y}^{2}\right)+E\left(S_{x}^{2}-S_{y}^{2}\right) .
$$

(6)式中求和限制符号表示仅对偶数 $k$ 求和.

超精细结构 Hamilton 量 


$$
H_{H F S}=H_{M}+H_{Q},
$$

其中磁超精细结构项

$$
H_{M}=a T^{1}(I) \cdot T^{1}(S)-10^{1 / 2} T^{1}(I) \cdot T^{1}\left(S, C^{2}\right),
$$

原子核电四极相互作用项(仅当 $I>1 / 2$ 时考虑)

$$
H_{\mathrm{Q}}=e T^{2}(Q) \cdot T^{2}(\nabla E) .
$$

外磁场 Zeeman 效应 Hamilton 量

$$
\begin{aligned}
H_{\mathrm{z}}= & B_{0}\left\{g_{s} \mu_{B} T_{0}^{1}(S)-\mu_{B} \sum_{k=0}^{2}(-1)^{k}[(2 k+1) / 3]^{1 / 2} T_{0}^{1}\left(g_{l}^{k}, S\right)\right. \\
& \left.\left.+\mu_{B} \sum_{k=0}^{2}(-1)^{k}[(2 k+1) / 3]^{1 / 2} T_{0}^{1}\left(g_{r}^{k}, N\right)-g_{N} \mu_{N} T_{0}^{1}(I)\right\}\right\},
\end{aligned}
$$

式中 $B_{0}$ 表示外磁场的磁感应强度.

以上(5) (12)式均参见文献[7].

与文献[1]相比, (4)式将分子转动离心畸变 Hamilton 量延伸到了 12 次方项, (7)式将电子自 旋-分子转动离心畸变 Hamilton 量延伸到了 8 次方项. 后面我们将看到, 高阶离心畸变项对于 准确计算非对称陀螺分子的能级尤其是高转动量子数能级起重要作用.

\section{2 标识分析涉及的其他问题}

非对称陀螺分子的电子自旋-轨道耦合很弱, 适用 Hund 耦合情形(b). 与文献[1]一样, 本 文采用完全耦合表象，即

$$
\boldsymbol{N}+\boldsymbol{S}=\boldsymbol{J}, \quad \boldsymbol{J}+\boldsymbol{I}=\boldsymbol{F}
$$

基矢形如

$$
\left|\eta N K_{a} S J F M_{F}\right\rangle,
$$

其中 $\eta$ 代表惟一地标识特定量子态所需的所有其他量子数, 如振动量子数和电子态标记; $K_{a}$ 是 $N$ 在惯量主轴 $a$ 上的投影.

针对特定的表象和基矢, 可建立 Hamilton 矩阵. Hamilton 矩阵的本征值即为分子能级. 但 完整的基矢空间是一个无穷维的 Hilbert 空间, 导致 Hamilton 矩阵维数亦为无穷大. 分子能级 的机器计算一般采取围绕待求解量子态在一定量子数范围内构造基矢子空间, 建立 Hamilton 子阵, 经对角化获得该量子态对应的能级 ${ }^{[1]}$.

由于特定量子态的能级通过将与基矢子空间对应的 Hamilton 子阵对角化求得，同一基矢 子空间中的基矢应具有与该特定量子态相同的宇称. 因此，本文采用宇称守恒基矢

$$
\left.|\eta N K m \pm\rangle=2^{-1 / 2}\right\rangle\left\{|\eta N K m\rangle \pm(-)^{N-K}|\eta N-K m\rangle\right\} \text {. }
$$

将 Hamilton 子阵对角化可求得相应量子态的本征值和本征矢. 但本征值须从对角化后 Hamilton子阵的对角元中选取, 究竟哪一个对角元是该量子态对应的能级, 这在非对称陀螺自 由基分子磁共振谱的标识分析过程中是一个难题. 对于处在外磁场中的自由基分子, 基矢 $\left|\eta N K_{a} S J I F M_{F}\right\rangle$ 中除 $N, K_{a}$ 和 $M_{F}$ 外其余的量子数严格来讲不再是好量子数. 亦即, 我们借以 标识特定量子态的基矢 $\left|\eta N K_{a} S J I F M_{F}\right\rangle$ 并不是 Hamilton 量真正的本征态. 对角化获得的本征 
矢方阵 $\boldsymbol{S}$ 列矢并不是由具有可观测意义的量子数组成, 无法从中直接看出与特定量子态的对 应关系.

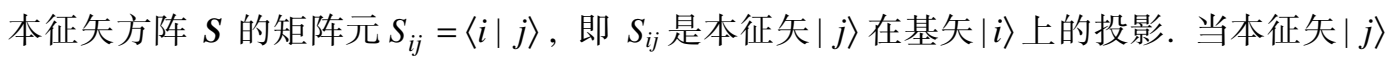
在基矢 $|i\rangle$ 上的投影足够大时，作为一种合理近似，可将基矢 $|i\rangle$ 对应为本征矢 $|j\rangle$. 在 $\boldsymbol{S}$ 方阵 中, 如果某一列矢 $S_{j}$ 中的最大元素为 $S_{i j}$, 而待求解量子态在基矢矩阵中正好处于第 $i$ 行, 则该 量子态对应的能级为对角化后的 Hamilton 子阵对角元 $D_{j j}$.

非对称陀螺分子电偶极跃迁的选择规则分 3 种类型:
A 型 $\Delta N=0, \pm 1 ; \Delta K_{a}$ 为偶整数; $\Delta K_{c}$ 为奇整数;
B 型 $\Delta N=0, \pm 1 ; \Delta K_{a}$ 为奇整数; $\Delta K_{c}$ 为奇整数;
C 型 $\Delta N=0, \pm 1 ; \Delta K_{a}$ 为奇整数; $\Delta K_{c}$ 为偶整数.

磁偶极跃迁的选择规则中 $\Delta K_{a}$ 和 $\Delta K_{c}$ 的奇偶性与之相反.

分子在 $a, b$ 态之间的电偶极跃迁强度可用不可约张量表示如下 ${ }^{[7]}$ :

$$
I_{a b}=\left(N_{a}-N_{b}\right) v_{a b} \sum_{p}\left|\left\langle a\left|T_{p}^{1}(\boldsymbol{\mu}) \cdot T_{-p}^{1}(\boldsymbol{E})\right| b\right\rangle\right|^{2},
$$

式中 $T^{1}(\boldsymbol{\mu})$ 代表分子电偶极矩算符, $T^{1}(\boldsymbol{E})$ 代表辐射场电矢量张量, $\sum T^{1}(\boldsymbol{\mu}) \cdot T^{1}(\boldsymbol{E})$ 代表它们 之间的 Stark 相互作用; $N_{a}, N_{b}$ 分别为 $a$ 态和 $b$ 态的分子布居数密度; $v_{a b}$ 为跃迁频率. 非对称陀 螺分子谱线的相对强度可由(16)式计算.

对非对称陀螺自由基分子的磁共振谱, 由于激光频率是固定的, 扫描的是磁场强度, 往往 需计算外磁场对分子谱线或能级的Zeeman 调制率. Zeeman 调制率指分子能级或跃迁频率对磁 场的变化率. 外磁场对跃迁频率的调制率等于上下两能级的调制率之差, 即

$$
\frac{\partial v_{a b}}{\partial B_{0}}=\frac{\partial E_{a}}{\partial B_{0}}-\frac{\partial E_{b}}{\partial B_{0}} .
$$

文献[8]中给出了如下公式:

$$
\frac{\partial E_{i}}{\partial p_{j}}=\left[S \cdot \frac{\partial H}{\partial p_{j}} \cdot S^{\mathrm{T}}\right]_{i i},
$$

式中 $E_{i}$ 代表 Hamilton 子阵的第 $i$ 个本征值, $p_{j}$ 代表第 $j$ 个分子参数, $\boldsymbol{S}$ 为本征矢方阵, $\partial H / \partial p_{j}$ 是 Hamilton 量对第 $j$ 个参数的导数. 这个公式很重要, 在求 Zeeman 调制率的时候要用到它, 在 作最小二乘拟合时也要用到它.

根据前述理论模型，我们编制了一套非对称陀螺自由基分子光谱的标识分析计算程序.

\section{$3{ }^{14} \mathrm{~N}^{16} \mathrm{O}_{2}$ 基振动态光谱的标识分析}

我们采用本文介绍的标识分析计算方法及程序对迄今为止已获得的 ${ }^{14} \mathrm{~N}^{16} \mathrm{O}_{2}$ 基振动态全部 1059 条实验谱线进行最小二乘拟合, 获得了目前最精确、最完整的 ${ }^{14} \mathrm{~N}^{16} \mathrm{O}_{2}$ 基振动态分子结构 参数, 其中 6 个高阶离心畸变参数是首次获得 ${ }^{\left[{ }^{2} 22\right]}$. 拟合所得参数与文献 [19]给出的参数相互 对照列于表 1 . 从表 1 中可以看出, 本文给出的分子结构参数与文献[19]相比, 精度有较大幅度 的提高, 部分参数的精度提高了一个量级以上, 整体拟合误差仅为文献[19]的三分之一.

表 2 给出了部分 ${ }^{14} \mathrm{~N}^{16} \mathrm{O}_{2}$ 基振动态纯转动跃迁的标识分析计算结果. 其中第 1 次计算未考 
表 $1{ }^{14} \mathrm{~N}^{16} \mathrm{O}_{2}$ 基振动态分子参数

\begin{tabular}{|c|c|c|c|c|c|c|c|}
\hline & 参数 $/ \mathrm{cm}^{-1}$ & 文献结果 ${ }^{a)}$ & 本文结果 & & 参数 $/ \mathrm{cm}^{-1}$ & 文献结果 ${ }^{a)}$ & 本文结果 \\
\hline \multirow{5}{*}{$10^{2}$} & $A$ & $8.00235555(38)^{\mathrm{b})}$ & $8.00235329(19)$ & & $T_{0}^{0}(\varepsilon)$ & $-0.1024411(14)$ & $-0.05914449(54)^{\mathrm{c}}$ \\
\hline & $(B+C) / 2$ & $0.422074670(24)$ & $0.422074590(13)$ & & $T_{0}^{2}(\varepsilon)$ & $0.1484500(20)$ & $-0.06060471(55)^{\mathrm{c})}$ \\
\hline & $(B-C) / 4$ & $0.58160507(45)$ & $0.581607009(62)$ & $10^{2}$ & $T_{ \pm 2}^{2}(\varepsilon)$ & $-0.171804(15)$ & $-0.1717821(68)$ \\
\hline & $B$ & 0.433706771 & $0.433706730(13)$ & & $\varepsilon_{a a}$ & 0.180353398 & $0.1803539(12)$ \\
\hline & $C$ & 0.410442568 & $0.410442450(13)$ & $10^{3}$ & $\varepsilon_{b b}$ & 0.257976 & $0.25760(76)$ \\
\hline $10^{2}$ & $\Delta_{K}$ & $0.2687877(41)$ & $0.2687755(13)$ & $10^{2}$ & $\varepsilon_{c c}$ & -0.3178112 & $-0.317804(76)$ \\
\hline $10^{4}$ & $\Delta_{N K}$ & $-0.196796(13)$ & $-0.1967902(35)$ & $10^{3}$ & $\Delta_{K}^{S}$ & $-0.17616(36)$ & $-0.17674(20)$ \\
\hline $10^{6}$ & $\Delta_{N}$ & $0.299263(37)$ & $0.2993048(89)$ & $10^{6}$ & $\Delta_{N K}^{S}+\Delta_{K N}^{S}$ & $0.586(27)$ & $0.646(14)$ \\
\hline $10^{5}$ & $\delta_{K}$ & $0.40324(51)$ & $0.405569(40)$ & $10^{5}$ & $\Delta_{N K}^{S}$ & $0.162(13)$ & $0.1801(59)$ \\
\hline $10^{7}$ & $\delta_{N}$ & $0.319450(42)$ & $0.3193142(42)$ & $10^{9}$ & $\Delta_{N}^{S}$ & $0.570(86)$ & $0.764(36)$ \\
\hline $10^{5}$ & $H_{K}$ & $0.30297(15)$ & $0.303038(16)$ & $10^{6}$ & $\delta_{K}^{S}$ & $0.347(62)$ & $0.439(29)$ \\
\hline $10^{7}$ & $H_{K N}$ & $-0.26960(29)$ & $-0.270169(78)$ & $10^{9}$ & $\delta_{K}^{S}$ & $0.241(43)$ & $0.251(21)$ \\
\hline $10^{10}$ & $H_{N K}$ & $0.205(24)$ & $0.29339(17)$ & $10^{6}$ & $H_{N}^{S}$ & $0.2955(59)$ & $0.2946(35)$ \\
\hline $10^{12}$ & $H_{N}$ & $0.298(12)$ & $0.2792(22)$ & $10^{9}$ & $L_{K}^{S}$ & $-0.346(25)$ & $-0.356(25)$ \\
\hline $10^{7}$ & $h_{K}$ & $0.246(10)$ & $0.29423(61)$ & $10^{2}$ & $a_{N}$ & $0.491297(61)$ & $0.491309(25)$ \\
\hline $10^{10}$ & $h_{K N}$ & $-0.257(32)$ & $-0.3790(34)$ & $10^{3}$ & $T_{0}^{2}(c)$ & $-0.36786(99)$ & $-0.36823(41)$ \\
\hline $10^{12}$ & $h_{N}$ & $0.1072(18)$ & $0.1057(17)$ & $10^{3}$ & $T_{ \pm 2}^{2}(c)$ & $-0.39228(41)$ & $-0.39250(21)$ \\
\hline $10^{8}$ & $L_{K}$ & $-0.5077(22)$ & $0.51103(15)$ & $10^{3}$ & $T_{a a}(c)$ & -0.7357293 & $-0.73646(82)$ \\
\hline $10^{10}$ & $L_{K K N}$ & $0.3485(13)$ & $0.3511(13)$ & $10^{2}$ & $T_{b b}(c)$ & 0.13287525 & $0.132966(65)$ \\
\hline $10^{12}$ & $L_{K N}$ & $0.103(10)$ & $0.1215(97)$ & $10^{5}$ & $T_{0}^{2}(\nabla E)$ & $0.720(76)$ & $0.738(23)$ \\
\hline $10^{14}$ & $L_{N N K}$ & & $-0.337(66)$ & $10^{4}$ & $T_{ \pm 2}^{2}(\nabla E)$ & $-0.1930(27)$ & $-0.1920(13)$ \\
\hline $10^{17}$ & $L_{N}$ & & $-0.736(229)$ & $10^{4}$ & $T_{a a}(\nabla E)$ & 0.14419 & $0.1476(46)$ \\
\hline $10^{11}$ & $l_{K}$ & & $-0.587(216)$ & $10^{4}$ & $T_{b b}(\nabla E)$ & -0.545052 & $-0.5441(39)$ \\
\hline $10^{12}$ & $l_{K N}$ & & $-0.111(20)$ & $10^{5}$ & $\sigma$ & 0.89 & 0.31 \\
\hline $10^{14}$ & $l_{N K}$ & & $0.611(109)$ & & & & \\
\hline $10^{17}$ & $l_{N}$ & & $-0.332(64)$ & & & & \\
\hline $10^{11}$ & $P_{K}$ & $0.847(13)$ & $0.867(13)$ & & & & \\
\hline $10^{14}$ & $Q_{K}$ & $-0.803(30)$ & $-0.843(30)$ & & & & \\
\hline
\end{tabular}

a) 取自文献[19]; b) 括号中的数字为标准差; c) 本文对自旋-转动球张量的定义与文献[19]有所不同 
表 $2{ }^{14} \mathrm{~N}^{16} \mathrm{O}_{2}$ 基振动态部分纯转动跃迁

\begin{tabular}{|c|c|c|c|c|c|c|c|c|c|c|c|c|c|c|}
\hline \multirow{2}{*}{$N^{\prime}$} & \multirow{2}{*}{$K_{a}^{\prime}$} & \multirow{2}{*}{$K_{c}^{\prime}$} & \multirow{2}{*}{$J^{\prime}$} & \multirow{2}{*}{$F^{\prime \prime}$} & \multirow{2}{*}{$N^{\prime \prime}$} & \multirow{2}{*}{$K_{a}^{\prime \prime}$} & \multirow{2}{*}{$K_{c}^{\prime \prime}$} & \multirow{2}{*}{$J^{\prime \prime}$} & \multirow{2}{*}{$F^{\prime \prime}$} & \multirow{2}{*}{ 频率 (观测值) } & \multicolumn{2}{|c|}{ 第 1 次计算 } & \multicolumn{2}{|c|}{ 第 2 次计算 ${ }^{a)}$} \\
\hline & & & & & & & & & & & 频率 & $\mathrm{O}-\mathrm{C}$ & 频率 & $\mathrm{O}-\mathrm{C}$ \\
\hline \multicolumn{15}{|c|}{ 微波光谱 b) } \\
\hline 3 & 1 & 3 & 2.5 & 3.5 & 2 & 0 & 2 & 1.5 & 2.5 & 300183.69 & 300183.81 & -0.12 & 300183.81 & -0.12 \\
\hline 4 & 1 & 3 & 3.5 & 4.5 & 4 & 0 & 4 & 3.5 & 3.5 & 229885.42 & 229885.36 & 0.06 & 229885.36 & 0.06 \\
\hline 6 & 1 & 5 & 5.5 & 6.5 & 6 & 0 & 6 & 5.5 & 5.5 & 233976.25 & 233976.33 & -0.08 & 233976.33 & -0.08 \\
\hline 8 & 1 & 7 & 7.5 & 8.5 & 8 & 0 & 8 & 7.5 & 7.5 & 239460.80 & 239460.76 & 0.04 & 239460.76 & 0.04 \\
\hline 10 & 1 & 9 & 10.5 & 9.5 & 10 & 0 & 10 & 9.5 & 9.5 & 246792.67 & 246792.79 & -0.12 & 246792.79 & -0.12 \\
\hline 13 & 2 & 12 & 12.5 & 13.5 & 14 & 1 & 13 & 14.5 & 14.5 & 289899.86 & 289899.90 & -0.04 & 289899.88 & -0.02 \\
\hline 14 & 1 & 13 & 14.5 & 13.5 & 14 & 0 & 14 & 13.5 & 13.5 & 265509.87 & 265509.98 & -0.11 & 265509.98 & -0.11 \\
\hline 15 & 2 & 14 & 14.5 & 15.5 & 16 & 1 & 15 & 16.5 & 16.5 & 229062.79 & 229062.88 & -0.09 & 229062.86 & -0.07 \\
\hline 16 & 1 & 15 & 16.5 & 15.5 & 16 & 0 & 16 & 15.5 & 15.5 & 277710.24 & 277710.32 & -0.08 & 277710.32 & -0.08 \\
\hline 18 & 2 & 16 & 18.5 & 17.5 & 19 & 1 & 19 & 18.5 & 18.5 & 274843.12 & 274842.97 & 0.15 & 274842.96 & 0.16 \\
\hline 20 & 2 & 18 & 20.5 & 19.5 & 21 & 1 & 21 & 20.5 & 20.5 & 242820.46 & 242820.49 & -0.03 & 242820.49 & -0.03 \\
\hline 22 & 2 & 20 & 22.5 & 21.5 & 23 & 1 & 23 & 22.5 & 22.5 & 213411.83 & 213411.75 & 0.08 & 213411.75 & 0.08 \\
\hline 31 & 3 & 29 & 31.5 & 30.5 & 32 & 2 & 30 & 31.5 & 31.5 & 274872.28 & 274872.36 & -0.08 & 274872.26 & 0.02 \\
\hline 33 & 3 & 31 & 33.5 & 33.5 & 34 & 2 & 32 & 33.5 & 34.5 & 212699.54 & 212699.56 & -0.02 & 212699.61 & -0.07 \\
\hline 36 & 3 & 33 & 35.5 & 34.5 & 37 & 2 & 36 & 36.5 & 35.5 & 239317.67 & 239317.53 & 0.14 & 239317.80 & -0.13 \\
\hline 46 & 2 & 44 & 45.5 & 46.5 & 45 & 3 & 43 & 44.5 & 45.5 & 196088.28 & 196089.74 & -1.46 & 196088.44 & -0.16 \\
\hline 51 & 4 & 48 & 50.5 & 49.5 & 52 & 3 & 49 & 51.5 & 50.5 & 213516.94 & 213512.90 & 4.04 & 213517.00 & -0.06 \\
\hline \multicolumn{15}{|c|}{ 远红外光谱 c) } \\
\hline 5 & 4 & 2 & 4.5 & 4.5 & 4 & 3 & 1 & 3.5 & 3.5 & 56.74668 & 56.74692 & -0.00024 & 56.74663 & 0.00005 \\
\hline 25 & 6 & 20 & 24.5 & 23.5 & 26 & 5 & 21 & 25.5 & 24.5 & 59.78830 & 59.79374 & -0.00544 & 59.78828 & 0.00002 \\
\hline 9 & 9 & 1 & 9.5 & 10.5 & 9 & 8 & 2 & 9.5 & 10.5 & 123.10842 & 123.22326 & -0.11484 & 123.10900 & -0.00058 \\
\hline 10 & 9 & 1 & 10.5 & 11.5 & 10 & 8 & 2 & 10.5 & 11.5 & 123.09897 & 123.21168 & -0.11271 & 123.09761 & 0.00136 \\
\hline 10 & 9 & 1 & 10.5 & 10.5 & 10 & 8 & 2 & 10.5 & 10.5 & 123.09897 & 123.21175 & -0.11278 & 123.09768 & 0.00129 \\
\hline 10 & 9 & 1 & 10.5 & 9.5 & 10 & 8 & 2 & 10.5 & 9.5 & 123.09897 & 123.21184 & -0.11287 & 123.09776 & 0.00121 \\
\hline 13 & 9 & 5 & 13.5 & 14.5 & 13 & 8 & 6 & 13.5 & 14.5 & 123.08578 & 123.19877 & -0.11299 & 123.08539 & 0.00039 \\
\hline 13 & 9 & 5 & 13.5 & 13.5 & 13 & 8 & 6 & 13.5 & 13.5 & 123.08578 & 123.19881 & -0.11303 & 123.08543 & 0.00035 \\
\hline 13 & 9 & 5 & 13.5 & 12.5 & 13 & 8 & 6 & 13.5 & 12.5 & 123.08578 & 123.19886 & -0.11308 & 123.08548 & 0.00030 \\
\hline
\end{tabular}


续表 2

\begin{tabular}{|c|c|c|c|c|c|c|c|c|c|c|c|c|c|c|}
\hline \multirow{2}{*}{$N^{\prime}$} & \multirow{2}{*}{$K_{a}^{\prime}$} & \multirow{2}{*}{$K_{c}^{\prime}$} & \multirow{2}{*}{$J^{\prime}$} & \multirow{2}{*}{$F^{\prime}$} & \multirow{2}{*}{$N^{\prime \prime}$} & \multirow{2}{*}{$K_{a}^{\prime \prime}$} & \multirow{2}{*}{$K_{c}^{\prime \prime}$} & \multirow{2}{*}{$J^{\prime \prime}$} & \multirow{2}{*}{$F^{\prime \prime}$} & \multirow{2}{*}{ 频率 (观测值) } & \multicolumn{2}{|c|}{ 第 1 次计算 } & \multicolumn{2}{|c|}{ 第 2 次计算 ${ }^{a)}$} \\
\hline & & & & & & & & & & & 频率 & $\mathrm{O}-\mathrm{C}$ & 频率 & $\mathrm{O}-\mathrm{C}$ \\
\hline 14 & 9 & 5 & 13.5 & 12.5 & 12.5 & 14 & 8 & 6 & 13.5 & 12.5 & 122.90305 & -0.11340 & 122.90333 & -0.00028 \\
\hline 14 & 9 & 5 & 13.5 & 13.5 & 14 & 8 & 6 & 13.5 & 13.5 & 122.90305 & 123.01650 & -0.11345 & 122.90338 & -0.00033 \\
\hline 14 & 9 & 5 & 13.5 & 14.5 & 14 & 7 & 6 & 13.5 & 14.5 & 122.90305 & 123.01655 & -0.11350 & 122.90343 & -0.00038 \\
\hline 15 & 9 & 7 & 14.5 & 13.5 & 15 & 8 & 8 & 14.5 & 13.5 & 122.91852 & 123.03159 & -0.11307 & 122.91876 & -0.00024 \\
\hline 15 & 9 & 7 & 14.5 & 14.5 & 15 & 8 & 8 & 14.5 & 14.5 & 122.91852 & 123.03163 & -0.11311 & 122.91880 & -0.00028 \\
\hline 15 & 9 & 7 & 14.5 & 15.5 & 15 & 8 & 8 & 14.5 & 15.5 & 122.91852 & 123.03167 & -0.11315 & 122.91884 & -0.00032 \\
\hline 16 & 9 & 7 & 15.5 & 14.5 & 16 & 8 & 8 & 15.5 & 14.5 & 122.93330 & 123.04617 & -0.11287 & 122.93365 & -0.00035 \\
\hline 16 & 9 & 7 & 15.5 & 15.5 & 16 & 8 & 8 & 15.5 & 15.5 & 122.93330 & 123.04621 & -0.11291 & 122.93368 & -0.00038 \\
\hline 16 & 9 & 7 & 15.5 & 16.5 & 16 & 8 & 8 & 15.5 & 16.5 & 122.93330 & 123.04625 & -0.11295 & 122.93372 & -0.00042 \\
\hline 18 & 9 & 9 & 17.5 & 16.5 & 18 & 8 & 10 & 17.5 & 16.5 & 122.96251 & 123.07452 & -0.11201 & 122.96267 & -0.00016 \\
\hline 18 & 9 & 9 & 17.5 & 17.5 & 18 & 8 & 10 & 17.5 & 17.5 & 122.96251 & 123.07455 & -0.11204 & 122.96270 & -0.00019 \\
\hline 18 & 9 & 9 & 17.5 & 18.5 & 18 & 8 & 10 & 17.5 & 18.5 & 122.96251 & 123.07458 & -0.11207 & 122.96273 & -0.00022 \\
\hline 20 & 9 & 11 & 20.5 & 21.5 & 20 & 8 & 12 & 20.5 & 21.5 & 123.11604 & 123.22735 & -0.11131 & 123.11625 & 0.00021 \\
\hline 20 & 9 & 11 & 20.5 & 20.5 & 20 & 8 & 12 & 20.5 & 20.5 & 123.11604 & 123.22736 & -0.11132 & 123.11627 & -0.00023 \\
\hline 20 & 9 & 11 & 20.5 & 19.5 & 20 & 8 & 12 & 20.5 & 19.5 & 123.11604 & 123.22739 & -0.11135 & 123.11629 & -0.00025 \\
\hline 22 & 9 & 13 & 21.5 & 20.5 & 22 & 8 & 14 & 21.5 & 20.5 & 123.02093 & 123.13127 & -0.11034 & 123.02100 & -0.00007 \\
\hline 22 & 9 & 13 & 21.5 & 21.5 & 22 & 8 & 14 & 21.5 & 21.5 & 123.02093 & 123.13129 & -0.11036 & 123.02102 & -0.00009 \\
\hline 22 & 9 & 13 & 21.5 & 22.5 & 22 & 8 & 14 & 21.5 & 22.5 & 123.02093 & 123.13131 & -0.11038 & 123.02104 & -0.00011 \\
\hline 24 & 9 & 15 & 23.5 & 22.5 & 24 & 8 & 16 & 23.5 & 22.5 & 123.05144 & 123.16071 & -0.10927 & 123.05136 & 0.00008 \\
\hline 24 & 9 & 15 & 23.5 & 23.5 & 24 & 8 & 16 & 23.5 & 23.5 & 123.05144 & 123.16073 & -0.10929 & 123.05138 & 0.00006 \\
\hline 24 & 9 & 15 & 23.5 & 24.5 & 24 & 8 & 16 & 23.5 & 24.5 & 123.05144 & 123.16075 & -0.10931 & 123.05140 & 0.00004 \\
\hline 31 & 9 & 23 & 31.5 & 31.5 & 31 & 8 & 24 & 31.5 & 31.5 & 123.24756 & 123.35283 & -0.10527 & 123.24734 & 0.00022 \\
\hline 31 & 9 & 23 & 31.5 & 32.5 & 31 & 8 & 24 & 31.5 & 32.5 & 123.24756 & 123.35283 & -0.10527 & 123.24734 & 0.00022 \\
\hline 31 & 9 & 23 & 31.5 & 30.5 & 31 & 8 & 24 & 31.5 & 30.5 & 123.24756 & 123.35284 & -0.10528 & 123.24735 & 0.00021 \\
\hline 33 & 9 & 25 & 32.5 & 31.5 & 33 & 8 & 26 & 32.5 & 31.5 & 123.20421 & 123.30842 & -0.10421 & 123.20423 & -0.00002 \\
\hline 33 & 9 & 25 & 32.5 & 32.5 & 33 & 8 & 26 & 32.5 & 32.5 & 123.20421 & 123.30844 & -0.10423 & 123.20425 & -0.00004 \\
\hline 33 & 9 & 25 & 32.5 & 33.5 & 33 & 8 & 26 & 32.5 & 33.5 & 123.20421 & 123.30845 & -0.10424 & 123.20426 & -0.00005 \\
\hline 36 & 9 & 27 & 35.5 & 34.5 & 36 & 8 & 28 & 35.5 & 34.5 & 123.26274 & 123.36393 & -0.10119 & 123.26185 & 0.00089 \\
\hline 36 & 9 & 27 & 35.5 & 35.5 & 36 & 8 & 28 & 35.5 & 35.5 & 123.26274 & 123.36394 & -0.10120 & 123.26187 & 0.00087 \\
\hline 36 & 9 & 27 & 35.5 & 36.5 & 36 & 8 & 28 & 35.5 & 36.5 & 123.26274 & 123.36395 & -0.10121 & 123.26387 & 0.00087 \\
\hline
\end{tabular}

a) 第 1 次计算未考虑高阶离心畸变项, 第 2 次计算考虑了; b) 取自文献[13], 跃迁频率单位为 $\mathrm{MHz} ; \mathrm{c})$ 取自文献[19], 跃迁频率单位为 $\mathrm{cm}^{-1}$ 
虑高阶离心畸变项, 第 2 次计算考虑了. 比较这两次计算的结果可知高阶离心畸变项对分子跃 迁频率的贡献. 虽然高阶离心畸变项对低转动量子数跃迁无显著影响, 但随着转动量子数特 别是 $K$ 的升高, 高阶离心畸变项对分子跃迁频率的贡献越来越大, 当 $K$ 为 9 时, 高阶离心畸变 项对分子跃迁频率的贡献可达 $0.1 \mathrm{~cm}^{-1}$ 左右. 这说明高阶离心畸变项对 ${ }^{14} \mathrm{~N}^{16} \mathrm{O}_{2}$ 基振动态高转 动量子数跃迁有显著影响, 不容忽视.

70 年代初 Curl 等人观测了 ${ }^{14} \mathrm{~N}^{16} \mathrm{O}_{2}$ 远红外激光磁共振谱, 并对其中部分谱线进行了成功的 标识分析, 但其中激光频率为 $964313.4 \mathrm{MHz}$, 高场部分 $(B>1 T)$ 的磁共振谱线一直未获标识 ${ }^{[22]}$. 采用表 1 给出的分子结构参数, 我们尝试着对这些谱线进行了标识分析. 首先, 我们计算出 ${ }^{14} \mathrm{~N}^{16} \mathrm{O}_{2}$ 在激光频率 $\pm 2 \mathrm{~cm}^{-1}$ 范围内的全部零场跃迁; 然后针对这些零场跃迁搜索所有可能的 磁共振跃迁, 根据这些可能的磁共振跃迁的磁场位置、谱线强度和 Zeeman 调制率确认标识结 果. 表 3 是我们对 ${ }^{14} \mathrm{~N}^{16} \mathrm{O}_{2}$ 在激光频率为 $964313.4 \mathrm{MHz}$ 的情况下的磁共振谱的标识结果. 对于 $6_{3,3} \longleftarrow 7_{2,6}$ 和 $5_{3,3} \longleftarrow 6_{2,4}$ 的磁共振跃迁, 本文的标识计算结果与文献[20, 22]完全一致. $39_{2,38} \leftarrow$ $39_{1,39}$ 的高转动量子数磁共振跃迁为本文首次成功标识.

表 $3{ }^{14} \mathrm{~N}^{16} \mathrm{O}_{2}$ 基振动态在激光频率为 $964313.4 \mathrm{MHz}$ 时的磁共振谱的标识

\begin{tabular}{rrrrrrrrrrrrr}
\hline $\mathrm{N}^{\prime}$ & $K_{a}^{\prime}$ & $K_{c}^{\prime}$ & \multicolumn{1}{c}{$M_{F}^{\prime}$} & $P^{\prime} \leftarrow N^{\prime \prime}$ & $K_{a}^{\prime \prime}$ & $K_{c}^{\prime \prime}$ & $M_{F}^{\prime \prime}$ & $P^{\prime \prime}$ & $H \times 10^{4} / \mathrm{T}^{\mathrm{a})}$ & $(\mathrm{O}-\mathrm{C}) \times 10^{4} / \mathrm{T}$ & $(\partial \nu / \partial B)^{\mathrm{b})}$ \\
\hline 6 & 3 & 3 & 2.5 & -1 & 7 & 2 & 6 & 3.5 & 1 & 2746.2 & 0.06 & 2.56 \\
6 & 3 & 3 & -4.5 & -1 & 7 & 2 & 6 & -5.5 & 1 & 4604.0 & -1.09 & 2.59 \\
5 & 3 & 3 & -1.5 & -1 & 6 & 2 & 4 & -2.5 & 1 & 5394.4 & 0.96 & -2.57 \\
5 & 3 & 3 & -4.5 & -1 & 6 & 2 & 4 & -5.5 & 1 & 6651.8 & 0.47 & -2.70 \\
39 & 2 & 38 & -38.5 & 1 & 39 & 1 & 39 & -38.5 & -1 & 10184.2 & 0.06 & 2.28 \\
39 & 2 & 38 & -35.5 & 1 & 39 & 1 & 39 & -36.5 & -1 & 10261.1 & 0.41 & 2.30 \\
39 & 2 & 38 & -31.5 & 1 & 39 & 1 & 39 & -31.5 & -1 & 10481.1 & -0.24 & 2.38 \\
39 & 2 & 38 & -25.5 & 1 & 39 & 1 & 39 & -26.5 & -1 & 10645.9 & -0.67 & 2.44 \\
39 & 2 & 38 & -19.5 & 1 & 39 & 1 & 39 & -19.5 & -1 & 10838.7 & -0.19 & 2.51 \\
39 & 2 & 38 & -9.5 & 1 & 39 & 1 & 39 & -10.5 & -1 & 11114.4 & 0.03 & 2.59 \\
39 & 2 & 38 & -6.5 & 1 & 39 & 1 & 39 & -7.5 & -1 & 11199.8 & 0.04 & 2.62 \\
39 & 2 & 38 & 14.5 & 1 & 39 & 1 & 39 & 15.5 & -1 & 11722.3 & 0.23 & 2.74 \\
\hline
\end{tabular}

a) $H$ 为磁场强度; b) 单位为 $10 \mathrm{GHz} / \mathrm{T}$

\section{4 结论}

本文系统阐述了非对称陀螺自由基分子光谱的标识分析工作涉及的一些关键理论问题, 在有效 Hamilton 量中最大限度地考虑了高阶离心畸变项, 并建立了有效的标识分析计算程序. 作为应用实例以及对本文理论模型的一种检验, 文中对 ${ }^{14} \mathrm{~N}^{16} \mathrm{O}_{2}$ 基振动态的零场和磁共振谱进 行了统一分析, 获得了迄今为止最精确、最完整的 ${ }^{14} \mathrm{~N}^{16} \mathrm{O}_{2}$ 基振动态分子结构参数. 对 ${ }^{14} \mathrm{~N}^{16} \mathrm{O}_{2}$ 基振动态的高转动量子数跃迁的标识分析表明: 在有效 Hamilton 量中引人高阶离心畸变项之 后, 计算结果与相应的实验数据吻合得十分完美; 高阶离心畸变项对 ${ }^{14} \mathrm{~N}^{16} \mathrm{O}_{2}$ 基振动态的高转 动量子数跃迁有显著影响, 不容忽视. 此外, 还采用新得到的 ${ }^{14} \mathrm{~N}^{16} \mathrm{O}_{2}$ 基振动态分子结构参数, 对 Curl 等人过去未能标识的 ${ }^{14} \mathrm{~N}^{16} \mathrm{O}_{2}$ 远红外激光磁共振谱进行了成功的标识分析, 发现这些谱 线都属于 ${ }^{14} \mathrm{~N}^{16} \mathrm{O}_{2}$ 基振动态的高转动量子数跃迁. 对 ${ }^{14} \mathrm{~N}^{16} \mathrm{O}_{2}$ 基振动态光谱进行标识分析的结果 确认了所述理论模型的准确性、可靠性和完备性, 同时表明考虑高阶离心畸变项对许多非对称 陀螺分子光谱的标识分析是完全必要的. 


\section{参考文献}

1 Sears T J. The calculation of the energy levels of an asymmetric top free radical in a magnetic field. Comput Phys Rep, 1984, 2: $1 \sim 32$

2 Davies P B, Liu Y, Liu Z. Far infrared LMR spectra of monobromomethyl radicals. Chem Phys Lett, 1993, 214: $305 \sim 309$

3 Nolte J, Wagner H G, Sears T J, et al. The far-infrared laser magnetic resonance spectrum of $\mathrm{CH}_{2} \mathrm{~F}$. J Mol Spectrosc, 1999, 195: 43 53

4 Sears T J. ASYTOP_ A program for detailed analysis of gas phase magnetic resonance spectra of asymmetric top molecules. Comput Phys Commun, 1984, 34: 123 133

5 Papousek D, Aliev M R. Molecular Vibrational Rotational Spectra. Prague: Academia, 1982. 72 73

6 Matsushima F, Nagase H, Nakauchi T, et al. Frequency measurement of pure rotational transitions of $\mathrm{H}_{2} \mathrm{O}$. J Mol Spectrosc, 1999, 193: 217 223

7 Bowater I C, Brown J M, Carrington A. Microwave spectroscopy of nonlinear free radicals. Proc R Soc Lond A, 1973, 333: $265 \sim 288$

8 Castellano S, Bothner-by A A. Analysis of NMR spectra by least squares. J Chem Phys, 1964, 41: $3863 \sim 3869$

9 Bird G R. Microwave spectrum of $\mathrm{NO}_{2}$. J Chem Phys, 1956, 25: 1040 1043

10 Bird G R, Baird J C, Jache A W, et al. Microwave spectrum of $\mathrm{NO}_{2}$ : Fine structure and magnetic coupling. J Chem Phys, 1964, 40: $3378 \sim 3390$

11 Lees R M, Curl R F, Baker J G. Millimeter-wavelength microwave spectrum of nitrogen dioxide. J Chem Phys, 1966, 45: 2037 2040

12 Baron P A, Godfrey P D, Harris D O. Microwave spectrum of $\mathrm{NO}_{2}$ at $70 \mathrm{GHz}$. J Chem Phys, 1974, 60: 3723 3724

13 Bowman W C, De Lucia F C. The millimeter and submillimeter spectrum of $\mathrm{NO}_{2}$. J Chem Phys, 1982, 77: 92 107

14 Semmoud-Monnanteuil N, Colmont J M, Perrin A, et al. New measurements in the millimeter-wave spectrum of $\mathrm{NO}_{2}$. J Mol Spectrosc, 1989, 134: 176 182

15 Baskakov O I, Moskienko M V, Dyubko S F. Submillimeter rotational spectrum of nitrogen dioxide. Opt Spectrosc (USSR), 1982, 53: 270 272

16 Tanaka T, English A D, Field R W, et al. Microwave optical double resonance of $\mathrm{NO}_{2}$ with a tunable $\mathrm{cw}$ dye laser. J Chem Phys, 1973, 59: 5217 5218

17 Brown J M, Steimle T C, Coles M E, et al. A determination of the spin-rotation parameters for $\mathrm{NO}_{2}$. J Chem Phys, 1981, 74: $3668 \sim 3672$

Carli B, Carlotti M, Mencaraglia F, et al. The pure rotation spectrum of nitrogen dioxide. Mol Phys, 1984, 51: 1505 1509

Perrin A, Flaud J M, Camy-Peyret C, et al. The far infrared spectrum of $\mathrm{NO}_{2}$. Mol Phys, 1988, 63: 791 810

Curl R F, Evenson K M, Wells J S. Laser magnetic spectrum of $\mathrm{NO}_{2}$ at $337 \mu \mathrm{m}$ and $311 \mu \mathrm{m}$. J Chem Phys, 1972, 56: $5143 \sim 5151$

Burch D S, Tanttila W H, Mizushima M. X-band ESR spectrum of nitrogen dioxide. J Chem Phys, 1974, 61: 1607 1612 